\title{
Modelos de regressão aleatória na seleção de codornas de corte para produção de ovos
}

\author{
Bruno Bastos Teixeira(1), Ricardo Frederico Euclydes ${ }^{(1)}$, Luciano Pinheiro da Silva ${ }^{(1)}$, \\ Robledo de Almeida Torres(1), Felipe Gomes da Silva ${ }^{(1)}$, Antonio Policarpo Souza Carneiro(1), \\ Helmut Gonçalves Lehner( ${ }^{(1)}$ e Rafael Bastos Teixeira ${ }^{(1)}$
}

\begin{abstract}
(1)Universidade Federal de Viçosa, Departamento de Zootecnia, Avenida Peter Henry Rolfs, s/nó, CEP $36570-000$ Viçosa, MG. E-mail: brunobatzootecnia@yahoo.com.br, rbaja@ufv.br, lucianozooufv@yahoo.com.br, rtorres@ufv.br, felipepheu@yahoo.com.br, policarpo.carneiro@gmail.com, helmut_lehner@hotmail.com, rafaelzootecnia@yahoo.com.br
\end{abstract}

Resumo - O objetivo deste trabalho foi avaliar modelos de regressão aleatória com diferentes ordens de polinômios de Legendre, quanto ao melhor ajuste para a produção de ovos de codornas de corte. Foram avaliados os grupos genéticos UFV1 e UFV2 de codornas de corte, de origens distintas, do programa de melhoramento genético da Universidade Federal de Viçosa. Determinou-se a produção semanal de ovos de 1.294 matrizes, da $6^{\mathrm{a}}$ até a $57^{\mathrm{a}}$ semana de idade, das quais 644 do grupo genético UFV1 e 650 do UFV2. Utilizou-se o modelo animal em regressão aleatória pelo programa Wombat e, para modelar as trajetórias das características com o tempo, aplicaram-se as funções polinomiais de Legendre. Foram feitas comparações pelo critério de informação de Akaike, critério de informação bayesiano de Schwarz, logaritmo da função de verossimilhança e teste da razão de verossimilhança. $\mathrm{O}$ modelo com $\mathrm{K}=3$, para efeitos fixos, $\mathrm{K}_{\mathrm{a}}=4$, para efeitos genéticos, e $\mathrm{K}_{\mathrm{c}}=4$, para efeito de ambiente, propicia melhor ajuste para ambas as linhagens, não provoca grandes alterações nos componentes de variância e fornece melhores estimativas de herdabilidade.

Termos para indexação: Coturnix coturnix, herdabilidade, polinômios de Legendre, Wombat.

\section{Random regression models in the selection of meat-type quails for egg production}

\begin{abstract}
The objective of this work was to evaluate random regression models with different orders of Legendre polynomials, as to the best fit for egg production of meat-type quails. UFV1 and UFV2 genetic groups of meat-type quails, from different origins, of the genetic breeding program of the Universidade Federal de Viçosa, Brazil, were evaluated. Egg production was determined weekly for 1,294 quails, from their $6^{\text {th }}$ to their $57^{\text {th }}$ week of age, of which 644 were from the genetic group UFV1, and 650 from UFV2. The animal model was used in random regression by Wombat software, and, for modeling variable trajectories in time, the Legendre's polynomial functions were applied. Comparisons were made by the Akaike's information criterion, the Bayesian information criterion of Schwarz, the logarithm of the likelihood function, and the likelihood ratio test. The model with $K=3$, for fixed effects, $K_{a}=4$, for genetic effects, and $K_{c}=4$, for the effect of environment provides the best fit, do not causes major changes in the variance components, and provides better estimates of heritability.
\end{abstract}

Index terms: Coturnix coturnix, heritability, Legendre's polynomials, Wombat.

\section{Introdução}

A predição de valores genéticos quanto à persistência de postura em aves de corte é uma característica de grande impacto econômico que deve ser considerada no processo de seleção, em melhoramento genético (Teixeira et al., 2012; Venturini et al., 2012). Os modelos animais tradicionais de repetibilidade e o multicaracterístico fornecem predições de valores genéticos para determinados pontos da curva de crescimento, enquanto que os modelos de regressão aleatória (MRA) permitem essas estimativas para quaisquer pontos da curva (Sarmento et al., 2008). Segundo Teixeira et al. (2012), os MRA fornecem estimativas pontuais de herdabilidade e permitem obter estimativas de parâmetros genéticos para períodos específicos da curva.

O uso de MRA na modelagem da produção de ovos, em codornas de corte, foi proposto por Bonafé et al. (2011). Os autores consideraram esses modelos adequados pelo fato de que não requerem qualquer 
pressuposição sobre a forma da curva ou a trajetória da característica com o tempo. Ao ajustar um MRA, implicitamente, ajusta-se uma função contínua, que permite descrever as mudanças genéticas e ambientais com o passar do tempo (Teixeira et al., 2012).

Em vários trabalhos (Akbaş et al., 2004; Sarmento et al., 2008), os polinômios ortogonais de Legendre têm sido utilizados como funções contínuas, os quais podem requerer ordens de ajustes diferentes para cada efeito aleatório contido no modelo de análise (Bonafé et al., 2011). Segundo Sarmento et al. (2010), a falta de um modelo apropriado, isto é, o desconhecimento da ordem de ajuste mais apropriada para a função empregada, pode influenciar erroneamente a partição da variância fenotípica e as variâncias atribuídas aos efeitos incluídos no modelo. Na literatura, não se encontram recomendações dessas ordens de ajuste, quando se trabalha com seleção no melhoramento genético de codornas de corte.

O objetivo deste trabalho foi avaliar modelos de regressão aleatória, com diferentes ordens de polinômios de Legendre, quanto ao melhor ajuste para a produção de ovos de codornas de corte.

\section{Material e Métodos}

Foram utilizados dados de quatro gerações de Coturnix coturnix, do programa de melhoramento de aves da Universidade Federal de Viçosa. Dois grupos genéticos de codornas de corte, de origens distintas, denominados UFV1 e UFV2, foram avaliados. Estudou-se a produção semanal de ovos de 1.294 matrizes, das quais 644 eram provenientes do grupo genético UFV1 e 650 do UFV2. As matrizes foram avaliadas individualmente, quanto ao número de ovos obtidos da $6^{\mathbf{a}}$ à $57^{\mathrm{a}}$ semana de vida, em que a $6^{\mathrm{a}}$ semana de vida correspondeu à $1^{1}{ }^{a}$ semana de produção.

As informações originais continham dados de 1.632 fêmeas. No entanto, foram eliminadas 338 codornas da análise: as que não produziram ovos até a $12^{\mathrm{a}}$ semana ou as que produziram menos do que 120 ovos até a $52^{\underline{a}}$ semana.

A análise genética da produção de ovos utilizou o modelo animal em regressão aleatória pelo programa Wombat (Meyer, 2007). O modelo proposto incluiu os efeitos fixos de geração, a trajetória média de produção (regressão fixa), além dos seguintes efeitos aleatórios: genético aditivo direto $\left(\mathrm{K}_{\mathrm{a}}\right)$, de ambiente permanente direto $\left(\mathrm{K}_{\mathrm{c}}\right)$ e residual $(\varepsilon)$. Para modelar a trajetória fixa, foram utilizados os polinômios de Legendre de ordem três (quadrático) ou quatro (cúbico). As trajetórias aleatórias foram modeladas com os polinômios ortogonais de Legendre, tendo-se variado as ordens de ajustes de $\mathrm{K}_{\mathrm{a}}$ e $\mathrm{K}_{\mathrm{c}}$, que foram de três (quadrático) ou quatro (cúbico). Estas ordens de ajustes foram escolhidas para obtenção de modelo mais parcimonioso, pois modelagens com altos graus polinomiais, em conjuntos de dados com desbalanceamento do número de registros, geram estimativas erráticas ao fim do período, em razão do fenômeno de Runge, descrito por de Boor (2001).

O modelo geral utilizado na avaliação da produção de ovos dos dois grupos genéticos é descrito a seguir:

$$
\mathrm{y}_{\mathrm{ij}}=\mathrm{F}+\sum_{\mathrm{m}=1}^{\mathrm{k}} \beta_{\mathrm{m}} \phi_{\mathrm{m}}+\sum_{\mathrm{m}=1}^{\mathrm{ka}} \beta_{\mathrm{m}} \phi_{\mathrm{m}}+\sum_{\mathrm{m}=1}^{\mathrm{kc}} \beta_{\mathrm{m}} \phi_{\mathrm{m}}+\varepsilon_{\mathrm{ij}}
$$

em que: $y_{i j}$ é a produção de ovos na semana $j$ da codorna i; F refere-se aos efeitos fixos de geração; $\beta_{\mathrm{m}}$ é o coeficiente de regressão fixo da produção, sobre o polinômio de Legendre $m$, com função quadrática ou cúbica; $\alpha_{\text {im }}$ e $\rho_{\text {im }}$ são respectivamente os coeficientes de regressão do efeito genético aditivo direto e o de ambiente permanente do animal, para a codorna i; K é a ordem de ajuste do polinômio de Legendre (três ou quatro), para se averiguar qual é a mais apropriada para o efeito fixo; $K_{a}$ e $K_{c}$ são as ordens de ajuste dos polinômios de Legendre (três ou quatro) utilizadas para se averiguar qual a mais apropriada para cada efeito aleatório; $\varnothing_{\mathrm{m}}$ é a função polinomial de Legendre da idade padronizada $(-1<$ idade $<1)$; e $\varepsilon_{\mathrm{ij}}$ denota o efeito aleatório residual. A estrutura de variâncias residuais foi modelada, tendo-se considerado a homogeneidade de variâncias com base em análises prévias.

O modelo geral indicado anteriormente, com suas pressuposições, pode ser descrito na forma matricial da seguinte maneira:

$$
\begin{gathered}
\mathrm{y}=\mathrm{Xb}+\mathrm{Z}_{1} \mathrm{a}+\mathrm{Z}_{2} \mathrm{c}+\varepsilon \\
\mathrm{E}\left[\begin{array}{c}
\mathrm{y} \\
\mathrm{a} \\
\mathrm{c} \\
\varepsilon
\end{array}\right]=\left[\begin{array}{c}
\mathrm{Xb} \\
0 \\
0 \\
0
\end{array}\right]
\end{gathered}
$$

e $\operatorname{Var}(\mathrm{a})=\mathrm{K}_{\mathrm{a}} \otimes \mathrm{A}, \operatorname{Var}(\mathrm{c})=\mathrm{K}_{\mathrm{c}} \otimes \mathrm{I}_{\mathrm{Nd}}$, e $\operatorname{Var}(\varepsilon)=\mathrm{R}$; em que: y é o vetor de observações referentes a $N_{d}$ codornas; $\mathrm{b}$ é o vetor que contém os efeitos fixos e os coeficientes $b_{m}$ da regressão fixa; a é o vetor $K_{a} \times N$ de coeficientes de regressão aleatória do efeito genético aditivo direto, em que $\mathrm{N}$ denota o número total de 
codornas na matriz dos numeradores do coeficiente de parentesco entre os indivíduos; c é o vetor $\mathrm{K}_{\mathrm{c}} \times \mathrm{N}_{\mathrm{d}}$ de coeficientes de regressão aleatória do efeito de ambiente permanente de animal; $\varepsilon$ é o vetor de erros aleatórios; $X$ refere-se à matriz de incidência dos coeficientes de regressão fixos e de efeitos fixos; $Z_{1}$ e $Z_{2}$ referemse respectivamente às matrizes de incidência dos coeficientes de regressão aleatória do efeito genético aditivo direto e o de ambiente permanente de animal; $\mathrm{K}_{\mathrm{a}}$ e $\mathrm{K}_{\mathrm{c}}$ são matrizes de variâncias e covariâncias entre os coeficientes de regressão aleatórios do efeito genético aditivo direto e o de ambiente permanente de animal, respectivamente; A é a matriz dos numeradores do coeficiente de parentesco entre os indivíduos; $\mathrm{I}_{\mathrm{Nd}} \mathrm{e}$ a matriz de identidade de dimensão $\mathrm{N}_{\mathrm{d}}$; $\otimes$ é o operador de produto direto; $\mathrm{R}$ é a matriz diagonal de variâncias residuais, ou seja, $R=\sigma_{\mathrm{e}}^{2}$.

Foram testados oito diferentes modelos (Tabela 1); em todos eles, os polinômios de Legendre de ordem três ou quatro foram utilizados para modelar tanto a trajetória fixa quanto a trajetória aleatória.

Para testar a diferença entre os modelos estudados e, assim, escolher o melhor modelo, foram feitas comparações pelo critério de informação de Akaike (AIC) (Akaike, 1974) e o critério de informação bayesiano de Schwarz (BIC) (Nuñes-Antón \& Zimmerman, 2000), pelos valores de logaritmo da função de verossimilhança $\left(\log _{\mathrm{e}} \mathrm{L}\right)$ e pelo teste da razão de verossimilhança (LRT), a 1\% de probabilidade.

$\mathrm{AIC}, \mathrm{BIC}$ e $\log _{\mathrm{e}} \mathrm{L}$ foram obtidos pelo programa Wombat (Meyer, 2007). A estatística LRT foi obtida pela seguinte expressão: $\mathrm{LRT}_{\mathrm{ij}}=2 \log _{\mathrm{e}} \mathrm{L}_{1}-2 \log _{\mathrm{e}} \mathrm{L}_{\mathrm{j}}$, em que: $\log _{e} L_{i}$ é o máximo da função de verossimilhança,

Tabela 1. Modelos de regressão aleatória com suas respectivas ordens dos polinômios de Legendre para o ajuste da trajetória fixa $(\mathrm{K})$ e das trajetórias aleatórias $\left(\mathrm{K}_{\mathrm{a}}\right.$ e $\left.\mathrm{K}_{\mathrm{c}}\right)$.

\begin{tabular}{lccc}
\hline Modelo & \multicolumn{3}{c}{ Ordens dos Polinômios } \\
\cline { 2 - 4 } & $\mathrm{K}$ & $\mathrm{K}_{\mathrm{a}}$ & $\mathrm{K}_{\mathrm{c}}$ \\
\hline 1 & 3 & 3 & 3 \\
2 & 3 & 3 & 4 \\
3 & 3 & 4 & 3 \\
4 & 3 & 4 & 4 \\
5 & 4 & 3 & 3 \\
6 & 4 & 3 & 4 \\
7 & 4 & 4 & 3 \\
8 & 4 & 4 & 4 \\
\hline
\end{tabular}

$\mathrm{K}$, ordem de ajuste para o efeito fixo; $\mathrm{K}_{\mathrm{a}}$, ordem de ajuste para o efeito aleatório genético aditivo direto; $\mathrm{K}_{\mathrm{c}}$, ordem de ajuste para o efeito aleatório de ambiente permanente direto. para o modelo completo $\mathrm{i}$; e $\log _{\mathrm{e}} \mathrm{L}_{\mathrm{j}}$ é o máximo da função de verossimilhança, para o modelo reduzido j. A estimativa LRT foi comparada com o valor do qui-quadrado $\left(\chi^{2}\right)$ tabelado, com v graus de liberdade e significância de $1 \%$, em que $\mathrm{v}$ é a diferença entre o número de parâmetros estimados pelos modelos completo e reduzido (aninhados). A hipótese de nulidade testada foi a de que os modelos completo e reduzido não diferiram entre si. Se LRT $>\chi_{\text {tab }}^{2}(n ; 0,0,1)$, o teste é significativo e o modelo completo fornece melhor ajuste em comparação ao modelo reduzido.

\section{Resultados e Discussão}

Os modelos de maiores ordens propiciaram valores maiores de $\log _{\mathrm{e}} \mathrm{L}$ e menores de $\mathrm{AIC}$ e $\mathrm{BIC}$, o que indica que estes apresentam melhor ajuste nas duas linhagens (Tabela 2). Com base nos resultados de $\log _{\mathrm{e}} \mathrm{L}$, AIC e BIC indicam que o modelo quatro - com $\mathrm{K}=3$ para os efeitos fixos, $\mathrm{K}_{\mathrm{a}}=4$ para efeitos genético aditivo direto e $\mathrm{K}_{\mathrm{c}}=4$ para efeito de ambiente permanente de animal - propiciou melhor ajuste para a produção de ovos. No entanto, esse modelo é o mais parametrizado. $\mathrm{O}$ teste LRT indicou que os modelos avaliados diferiram estatisticamente entre si, ou seja, o modelo completo apresentou melhor ajuste em comparação ao modelo reduzido. Em ambas as linhagens, houve tendência de maior ajuste quando se aumentou o número de parâmetros dos modelos, e o melhor modelo foi o que apresentou ordem quatro, quanto ao efeito aditivo, e quatro quanto ao ambiente permanente. Quando não há superioridade significativa no ajuste entre o modelo 8 e o modelo 4 , opta-se pelo modelo mais parcimonioso de melhor ajuste pelos critérios analisados.

Resultados semelhantes foram obtidos por Silva et al. (2013), que estudaram o MRA quanto à produção de ovos em codornas e verificaram que a utilização de um modelo com 21 parâmetros e com os polinômios de Legendre de ordem três, para modelar a trajetória fixa, e de ordem quatro, para modelar as trajetórias aleatórias, proporcionaram melhor ajuste quanto à produção de ovos. Teixeira et al. (2012) verificaram que o modelo com 33 parâmetros, com $\mathrm{K}_{\mathrm{a}}=5$, para os efeitos genéticos aditivos diretos, e $\mathrm{K}_{\mathrm{c}}=5$, para o ambiente permanente de animal, proporcionou um melhor ajuste para modelar a trajetória de crescimento de codornas de corte do grupo genético UFV1. Assim, maiores ordens para o ajuste dos efeitos proporcionam melhores resultados. Para codornas do grupo genético 
UFV2, os autores verificaram que o modelo com 26 parâmetros, com $\mathrm{K}_{\mathrm{a}}=3$, para os efeitos aleatórios genéticos aditivos diretos, e $\mathrm{K}_{\mathrm{c}}=5$, para o ambiente permanente de animal, proporcionou um melhor ajuste. Akbaş et al. (2004) trabalharam com ganho de peso em codornas e verificaram melhor ajuste com um modelo de ordem seis. Este resultado indica que as maiores ordens poderiam proporcionar melhores resultados no ajuste dos efeitos, em modelagens de trajetórias de crescimento em codornas.

Sarmento et al. (2008) compararam modelos de regressão aleatória, para caprinos leiteiros, e observaram que os valores do $\log _{\mathrm{e}} \mathrm{L}$, AIC e BIC apontaram para a seleção de modelos com ordens mais altas; entretanto, os autovalores associados às matrizes de covariâncias, entre os coeficientes de regressão, indicaram a possibilidade de redução da dimensionalidade. No presente trabalho, o quarto autovalor, que representa o efeito genético aditivo em ambas as linhagens, tem contribuição muito próxima de zero, e o aumento de ordem polinomial não contribuiria para a explicação da trajetória dos valores genéticos (Tabela 3). Silva et al. (2013) relatam que, neste caso, as altas ordens de ajuste proporcionaram estimativas de variâncias genéticas, correlações genéticas e de ambiente permanente não condizentes com o fenômeno biológico estudado, e um modelo mais parcimonioso seria suficiente para ajustar as variâncias nos dados.

Verificou-se que o coeficiente de regressão 2 apresentou a maior variância, tanto para os efeitos genéticos aditivos diretos, quanto para os efeitos de ambiente permanente de animal, no modelo quatro, para os dois grupos genéticos (Tabela 3). Os autovalores podem ser uma ferramenta importante na escolha da ordem do polinômio a ser utilizado. Contudo, Legarra et al. (2004) chamam atenção ao fato de que a redução da dimensionalidade, em razão da eliminação de autovalores próximos de zero, não é indicada em todos os casos, uma vez que a adoção desse critério pode resultar em modelagem inadequada. Em ambas as linhagens, houve maior decréscimo da contribuição dos autovalores quanto ao aumento da ordem polinomial do efeito aditivo, e os últimos autovalores tiveram contribuição próxima de zero, diferentemente do efeito de ambiente permanente, que foi mais significativo nas duas linhagens.

Para o grupo UFV1, as estimativas de variância genética aditiva, as de ambiente permanente e a fenotípica foram da baixa a alta magnitude, para o modelo quatro, e oscilaram de 0,05 a 0,34 , de 0,53

Tabela 2. Ordem do polinômio (OP) para os efeitos fixos $(\mathrm{K})$, genético aditivo direto $\left(\mathrm{K}_{\mathrm{a}}\right)$ e de ambiente permanente $\left(\mathrm{K}_{\mathrm{c}}\right)$, número de parâmetros (NP), logaritmo da função de verossimilhança ( $\log _{\mathrm{e}} \mathrm{L}$ ), critério de informação de Akaike (AIC), critério de informação bayesiano (BIC) e teste da razão de verossimilhança (LRT), para os modelos de avaliação da produção de ovos em codornas de corte.

\begin{tabular}{|c|c|c|c|c|c|c|c|c|c|}
\hline \multirow[t]{2}{*}{ Modelo } & \multicolumn{3}{|c|}{$\mathrm{OP}$} & \multirow[t]{2}{*}{ NP } & \multirow[t]{2}{*}{$\log _{e} L$} & \multirow[t]{2}{*}{$\mathrm{AIC}$} & \multirow[t]{2}{*}{$\mathrm{BIC}$} & \multirow[t]{2}{*}{ LRT } & \multirow[t]{2}{*}{ Comparações $^{(1)}$} \\
\hline & $\mathrm{C}$ & $\mathrm{K}_{\mathrm{a}}$ & $\mathrm{K}_{\mathrm{c}}$ & & & & & & \\
\hline & & & & & & & UFV1 & & \\
\hline 1 & 3 & 3 & 3 & 13 & $-39.536,99$ & $79.099,99$ & $79.210,02$ & $1.277,67 * *$ & $(1-4)$ \\
\hline 2 & 3 & 3 & 4 & 17 & $-38.904,84$ & $77.843,71$ & $77.987,59$ & $13,39 * *$ & $(2-4)$ \\
\hline 3 & 3 & 4 & 3 & 17 & $-38.931,65$ & $77.897,33$ & $78.041,21$ & $67,01 * *$ & $(3-4)$ \\
\hline 4 & 3 & 4 & 4 & 21 & $-38.898,15$ & $77.838,33$ & $78.016,06$ & - & - \\
\hline 5 & 4 & 3 & 3 & 13 & $-39.536,99$ & $79.099,99$ & $79.210,02$ & $1.277,63 * *$ & $(5-8)$ \\
\hline 6 & 4 & 3 & 4 & 17 & $-38.904,84$ & $77.843,71$ & $77.987,59$ & $13,34 * *$ & $(6-8)$ \\
\hline 7 & 4 & 4 & 3 & 17 & $-38.931,50$ & $77.897,03$ & $78.040,90$ & $66,66 * *$ & $(7-8)$ \\
\hline \multirow[t]{2}{*}{8} & 4 & 4 & 4 & 21 & $-38.898,17$ & $77.838,37$ & $78.016,11$ & - & - \\
\hline & & & & & & & UFV2 & & \\
\hline 1 & 3 & 3 & 3 & 13 & $-40.563,53$ & $81.153,08$ & $81.262,99$ & $1.596,23^{* *}$ & - \\
\hline 2 & 3 & 3 & 4 & 17 & $-40.014,45$ & $80.062,91$ & $80.206,63$ & $498,05 * *$ & - \\
\hline 3 & 3 & 4 & 3 & 17 & $-39.802,22$ & $79.638,47$ & $79.782,19$ & $73,61 * *$ & - \\
\hline 4 & 3 & 4 & 4 & 21 & $-39.765,42$ & $79.572,87$ & $79.750,40$ & - & - \\
\hline 5 & 4 & 3 & 3 & 13 & $-40.563,53$ & $81.153,08$ & $81.262,99$ & $1.596,23 * *$ & - \\
\hline 6 & 4 & 3 & 4 & 17 & $-39.777,34$ & $79.588,71$ & $79.732,43$ & $23,85 * *$ & - \\
\hline 7 & 4 & 4 & 3 & 17 & $-39.796,36$ & $79.626,75$ & $79.770,47$ & $62,31 * *$ & - \\
\hline 8 & 4 & 4 & 4 & 21 & $-39.765,42$ & $79.572,87$ & $79.750,40$ & - & - \\
\hline
\end{tabular}

**Significativo a $1 \%$ de probabilidade. ${ }^{(1)}$ Comparações entre os modelos (entre parênteses), pelo teste LRT. 
a 4,29 e de 3,63 a 7,38 (Figura 1 A). Para UFV2, essas estimativas foram de média a alta magnitude e variaram de 0,12 a 1,75, de 0,66 a 4,04 e de 4,04 a 8,67 (Figura 1 B). Resultados semelhantes foram obtidos nos estudos de Akbaş et al. (2004), Dionello et al. (2008) e Bonafé et al. (2011), sobre a avaliação genética de postura de linhagens de codornas de corte, o que confirma a possibilidade de se usar o modelo quatro, por ser mais parcimonioso e não causar grandes alterações nas estimativas dos componentes de variância. Segundo Legarra et al. (2004), modelos mais parcimoniosos devem ser preferidos, pois um ajuste perfeito aumenta a demanda computacional (memória e capacidade de processamento) e a suscetibilidade a erros numéricos.

As estimativas de herdabilidade pelo ajuste do modelo quatro, para ambos os grupos genéticos, foram de baixa magnitude e variaram de 0,01 a 0,21 (Figura 2). Os outros modelos propostos (Sarmento et al., 2010; Bonafé et al., 2011; Teixeira et al., 2012) apresentaram estimativas de herdabilidade entre 0,01 a 0,5 , também de baixa magnitude. Esses resultados reforçam a possibilidade de se optar pelo modelo quatro, que é mais parcimonioso.

Tabela 3. Estimativas de variâncias (diagonal), covariâncias (abaixo da diagonal) e correlações (acima da diagonal) de cada grupo genético, entre os coeficientes de regressão aleatória (1, 2, 3 e 4) e os autovalores da matriz dos coeficientes, para o modelo quatro, para a produção de ovos.

\begin{tabular}{|c|c|c|c|c|c|c|c|c|c|}
\hline \multicolumn{5}{|c|}{ UFV1 } & \multicolumn{5}{|c|}{ UFV2 } \\
\hline 1 & 2 & 3 & 4 & Autovalor (\%) & 1 & 2 & 3 & 4 & Autovalor (\%) \\
\hline & \multicolumn{9}{|c|}{ Efeito genético aditivo direto $\left(\mathrm{K}_{\mathrm{a}}=4\right)$} \\
\hline 0,16046 & 0,92390 & 0,97080 & 0,95270 & 92,15 & 0,34790 & 0,56740 & $-0,6095$ & $-0,52740$ & 65,80 \\
\hline 0,35346 & 0,91221 & 0,84870 & 0,79110 & 7,70 & 0,22821 & 0,46499 & $-0,1515$ & $-0,19890$ & 27,42 \\
\hline 0,36057 & 0,75160 & 0,85967 & 0,99410 & 0,14 & $-0,23098$ & $-0,06634$ & 0,41274 & 0,98640 & 7,07 \\
\hline \multirow[t]{2}{*}{0,23040} & 0,45614 & 0,55642 & 0,36447 & 0,00 & $-0,23523$ & $-0,10257$ & 0,47916 & 0,57176 & 0,01 \\
\hline & \multicolumn{9}{|c|}{ Efeito de ambiente permanente $\left(\mathrm{K}_{\mathrm{c}}=4\right)$} \\
\hline 3,11470 & 0,80210 & 0,3320 & 0,18060 & 70,54 & 3,87910 & 0,72620 & 0,33880 & 0,38520 & 73,86 \\
\hline 2,53580 & 3,20900 & 0,6900 & 0,40880 & 22,85 & 2,95610 & 4,27190 & 0,79390 & 0,64030 & 20,60 \\
\hline 0,94754 & 1,99870 & 2,6146 & 0,76260 & 4,69 & 1,35860 & 3,34060 & 4,14500 & 0,79320 & 3,93 \\
\hline 0,32657 & 0,75044 & 1,2636 & 1,05020 & 1,91 & 0,81585 & 1,42330 & 1,73670 & 1,15660 & 1,62 \\
\hline
\end{tabular}
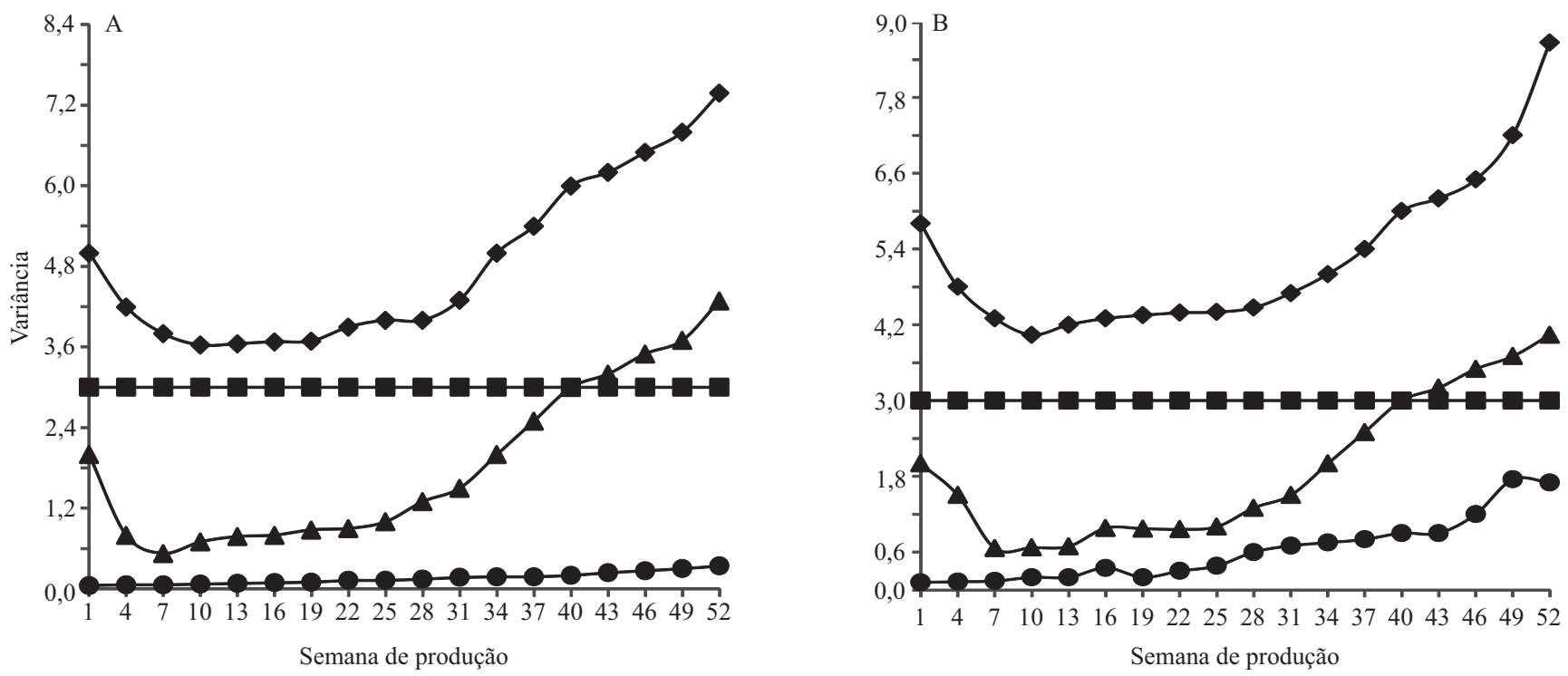

$\longrightarrow$ - Variância de ambiente permanente $\longrightarrow$ - Variância residual $\longrightarrow$ Variância genética aditiva

$\multimap$ Variância fenotípica

Figura 1. Variâncias genéticas aditivas, de ambiente permanente, fenotípicas e residuais, obtidas para o modelo de regressão aleatório de melhor ajuste à produção semanal de ovos. A, linhagem UFV1; B, linhagem UFV2. 

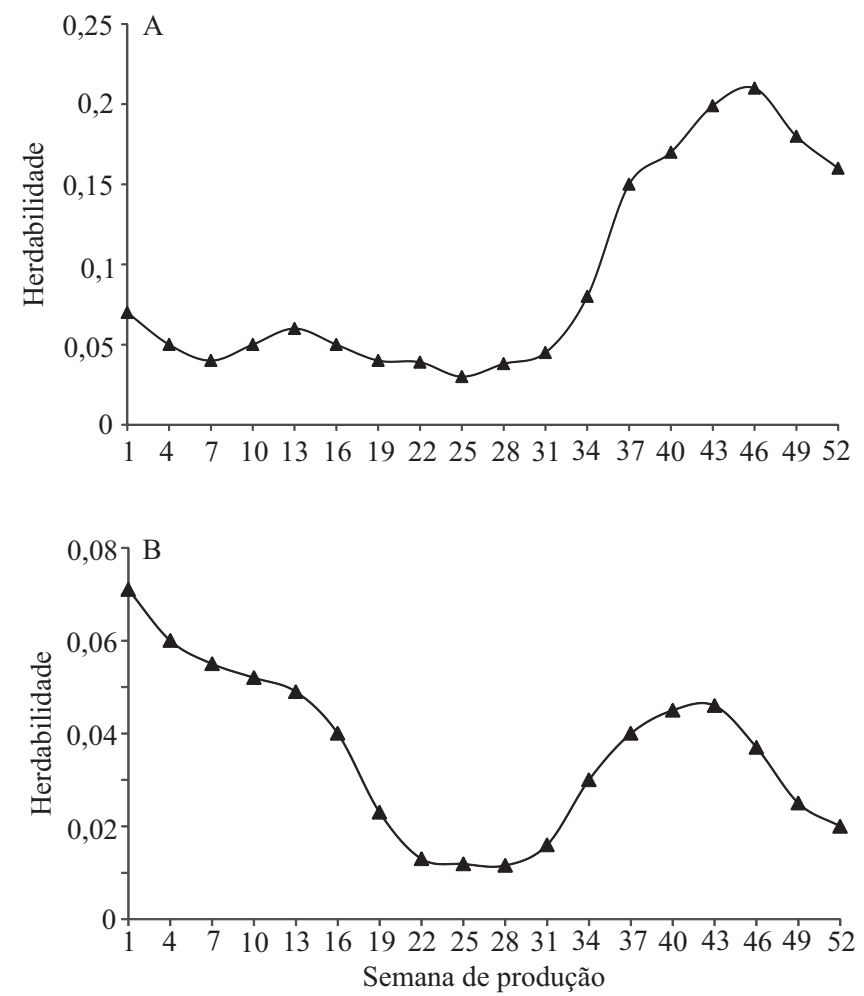

Figura 2. Estimativas de herdabilidade para o modelo de regressão aleatória de melhor ajuste à produção semanal de ovos; A, linhagem UFV1; B, linhagem UFV2.

\section{Conclusões}

1. O modelo com função polinomial de Legendre de ordem três, para os efeitos fixos, e de ordem quatro, para os efeitos aleatórios genéticos aditivos diretos e de ambiente permanente de animal, pode ser utilizado para descrever as trajetórias das produções de ovos de codornas de corte.

2. Este modelo não provoca grandes alterações nos componentes de variância e fornece melhores estimativas de herdabilidade para ambos os grupos genéticos.

\section{Referências}

AKAIKE, H. A new look at the statistical model identification. IEEE Transactions on Automatic Control, v.19, p.716-723, 1974. DOI: 10.1109/TAC.1974.1100705.

AKBAŞ, Y.; TAKMA, Ç.; YAYLAK, E. Genetic parameters for quail body weights using a random regression model. South
African Journal of Animal Science, v.34, p.104-109, 2004. DOI: 10.4314/sajas.v34i2.3813.

BONAFÉ, C.M.; TORRES, R. de A.; SARMENTO, J.L.R.; SILVA, L.P. da; RIBEIRO, J.C.; TEIXEIRA, R.B.; SILVA, F.G. da; SOUSA, M.F. Modelos de regressão aleatória para descrição da curva de crescimento de codornas de corte. Revista Brasileira de Zootecnia, v.40, p.765-771, 2011. DOI: 10.1590/S1516-35982011000400009.

BOOR, C. de. A practical guide to splines. $2^{\text {nd }}$ ed. New York: Springer, 2001. (Applied mathematics sciences, 27).

DIONELLO, N.J.L.; CORREA, G.S.S.; SILVA, M.A.; CORREAA, A.B.; SANTOS, G.G. Estimativas da trajetória genética do crescimento de codornas de corte utilizando modelos de regressão aleatória. Arquivo Brasileiro de Medicina Veterinária e Zootecnia, v.60, p.454-460, 2008. DOI: 10.1590/S0102-09352008000200027.

LEGARRA, A.; MISZTAL, I.; BERTRAND, J.K. Constructing covariance functions for random regression models for growth in Gelbvieh beef cattle. Journal of Animal Science, v.82, p.1564-1571, 2004.

MEYER, K. WOMBAT: a tool for mixed model analyses in quantitative genetics by REML. Journal of Zhejiang University Science-B, v.8, p.815-821, 2007. DOI: 10.1631/jzus.2007.B0815.

NÚÑEZ-ANTON, V.; ZIMMERMAN, D.L. Modeling nonstationary longitudinal data. Biometrics, v.56, p.699-705, 2000. DOI: 10.1111/j. 0006-341X.2000.00699.x.

SARMENTO, J.L.R.; ALBUQUERQUE, L.G. de; TORRES, R. de A.; RODRIGUES, M.T.; LOPES, P.S.; REIS FILHO, J.C. Comparação de modelos de regressão aleatória para estimação de parâmetros genéticos em caprinos leiteiros. Revista Brasileira de Zootecnia, v.37, p.1788-1796, 2008. DOI: 10.1590/S1516-35982008001000011.

SARMENTO, J.L.R.; TORRES, R. de A.; LÔBO, R.N.B.; ALBUQUERQUE, L.G. de; SOUSA, W.H. de; SOUSA, J.E.R. de. Modelos de regressão aleatória na avaliação genética do crescimento de ovinos da raça Santa Inês. Revista Brasileira de Zootecnia, v.39, p.1723-1732, 2010. DOI: 10.1590/S1516-35982010000800014.

SILVA, L.P.; RIBEIRO, J.C.; LEITE, C.D.S.; SOUSA, M.F.; BONAFÉ, C.M.; CAETANO, G.C.; CRISPIM, A.C.; TORRES, R.A. Evaluation of alternative schemes for recording body weights in meat-type quails by using random regression. Genetics and Molecular Research, v.12, p.1533-1539, 2013. DOI: 10.4238/2013. May.13.6.

TEIXEIRA, B.B.; EUCLYDES, R.F.; TEIXEIRA, R.B.; SILVA, L.P. da; TORRES, R. de A.; LEHNER, H.G.; CAETANO, G. da C.; CRISPIM, A.C. Modelos de regressão aleatória para avaliação da curva de crescimento em matrizes de codorna de corte. Ciência Rural, v.42, p.1641-1647, 2012. DOI: 10.1590/S0103-84782012000900020.

VENTURINI, G.C.; GROSSI, D.A.; RAMOS, S.B.; CRUZ, V.A.R.; SOUZA, C.G.; LEDUR, M.C.; EL FARO, L.; SCHMIDT, G.S.; MUNARI, D.P. Estimation of genetic parameters for partial egg production periods by means of random regression models. Genetics and Molecular Research, v.11, p.1819-1829, 2012. DOI: 10.4238/2012.July.10.18. 